\title{
Real-Time Interactive Viewing of 4D Kinematic MR Joint Studies
}

\author{
Heinrich Schulz ${ }^{1}$, Kirsten Meetz ${ }^{1}$, Clemens Bos ${ }^{2}$, Daniel Bystrov ${ }^{1}$, and Thomas Netsch ${ }^{1}$ \\ ${ }^{1}$ Philips Research Laboratories, Sector Technical Systems, \\ Roentgenstrasse 24-26, D-22335 Hamburg, Germany \\ heinrich.schulz@philips.com \\ ${ }^{2}$ Philips Medical Systems \\ Veenpluis 4-6, 5680 DA Best, The Netherlands
}

\begin{abstract}
Assessment of soft tissue in normal and abnormal joint motion today gets feasible by acquiring time series of 3D MRI images. However, slice-byslice viewing of such 4D kinematic images is cumbersome, and does not allow appreciating the movement in a convenient way. Simply presenting slice data in a cine-loop will be compromised by through-plane displacements of anatomy and "jerks" between frames, both of which hamper visual analysis of the movement. To overcome these limitations, we have implemented a demonstrator for viewing 4D kinematic MRI datasets. It allows to view any user defined anatomical structure from any viewing perspective in real-time. Smoothly displaying the movement in a cine-loop is realized by image post processing, fixing any user defined anatomical structure after image acquisition.
\end{abstract}

\section{Introduction}

Starting in the late eighties, MRI has been used for imaging of moving joints [1,2]. Most approaches were based on fast 2D imaging, which limited the study to a single predefined view of a few anatomical structures. In addition, in order to keep the anatomy of interest stable in the imaging plane, devices are needed to restrain a part of the joint [3], thereby limiting the freedom of movement. As a result pathological behavior in the kinematics of the investigated structures might be alleviated or even not show up at all.

MRI is capable of acquiring time series of 3D images, which gives a 4D examination that can be used for kinematic joint imaging of an unrestricted movement. Furthermore, 4D data acquisition greatly simplifies scan planning, because it obviates the need for defining and tracking the diagnostically relevant $2 \mathrm{D}$ view during image acquisition. However, slice-by-slice viewing of the 4D images is cumbersome, and does not allow to appreciate the movement. Simply presenting slice data in a cine-loop will be compromised by through-plane displacements of anatomy and "jerks" between frames, both of which hamper visual analysis of the movement.

We have implemented a demonstrator for viewing 4D kinematic MRI datasets that addresses the following requirements: 1) The motion of any anatomy can be viewed from any perspective. 2) The user may define that object or region of interest, e.g. the distal femur in kinematic joint imaging of the knee, which should remain fixed in the 
viewing plane during the movement. 3) The user interaction is reflected immediately in the viewing plane.

\section{Method}

\subsection{Viewing Workstation}

The proposed viewing workstation for 4D kinematic studies comprises two main steps of user interaction: the definition of the anatomical structure to be stabilized in visualization and real-time interaction with the video component in order to refine the selection of the anatomical structure of choice as well as to control imaging parameters like the zoom factor. Fig 1 shows a typical configuration of the user interface.

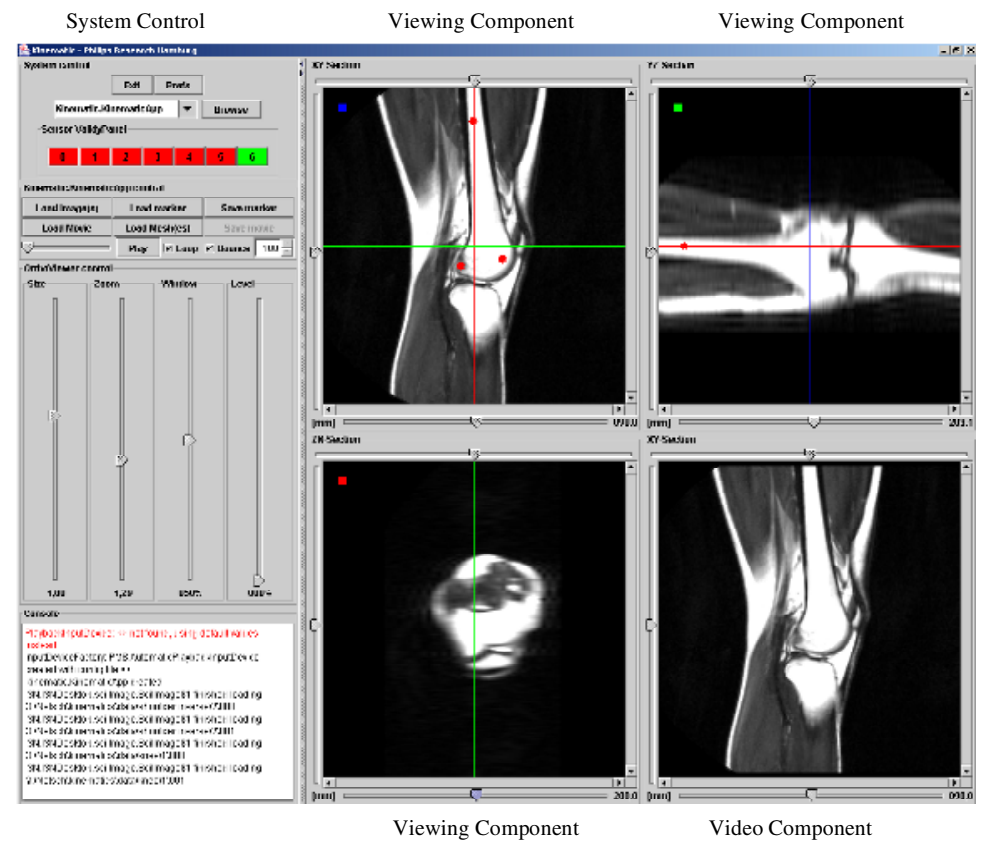

Fig. 1. User interface of the kinematic viewing station

\subsubsection{Definition of Anatomical Structure}

The viewing workstation allows the inspection of 4D data sets in three orthogonal viewing planes. It comprises the well-known 3D Orthoviewer functionalities depicting one 3D data set. In addition it allows switching between $\mathrm{n} 3 \mathrm{D}$ data sets retaining a previously defined viewing perspective with respect to the scanner coordinate system. Viewing functionality is implemented to enable the user to navigate through the data sets by translation and rotation, to scale the data set, and to manipulate window and level. Thus the entire 4D data set can be inspected from any user selected perspective. The definition of an anatomical structure of interest is done in order to fix this 
structure for visualization in cine mode. The fixation can be applied to virtually arbitrary regions and comprises two steps:

First, the movement of an anatomical structure through all 3D data sets is calculated by motion tracking. Therefore, the object or region of interest is defined interactively by setting reference points in one of the $3 \mathrm{D}$ data sets. The user has to set a minimum of 3 non-collinear reference points by clicking at any appropriate point within the viewing component. These points are propagated to all other data sets by using a motion estimation method. The propagation of the selected points for all data sets is calculated in advance by an elastic registration approach. The tracking of the object or region of interest is calculated by rigid point-based registration of the $n$ sets of reference points using singular value decomposition [4]. Both methods are described in Section 2.2 in detail.

Secondly, the inverse of the transformation defined by the tracking is used to align the $3 \mathrm{D}$ data sets, such that the defined objects or regions of interest remain stable when presented in cine loop.

\subsubsection{Real-Time Interaction}

The anatomical structures to be inspected for medical examination do not necessarily match those being fixated. When the kinematics data set is presented in cine mode the ability to manipulate the visualized part in real-time is provided to the user. For that purpose, the previously described user-interactions of defining a viewing perspective and defining an anatomical structure to be stabilized are combined appropriately. The combination of image transformations is applied to each of $n$ 3D data sets, showing the stabilized anatomy in the defined viewing perspective in cine mode. Every user interaction concerning viewing aspects like the selection of reference points or the change of the viewing perspective is reflected immediately in the video component.

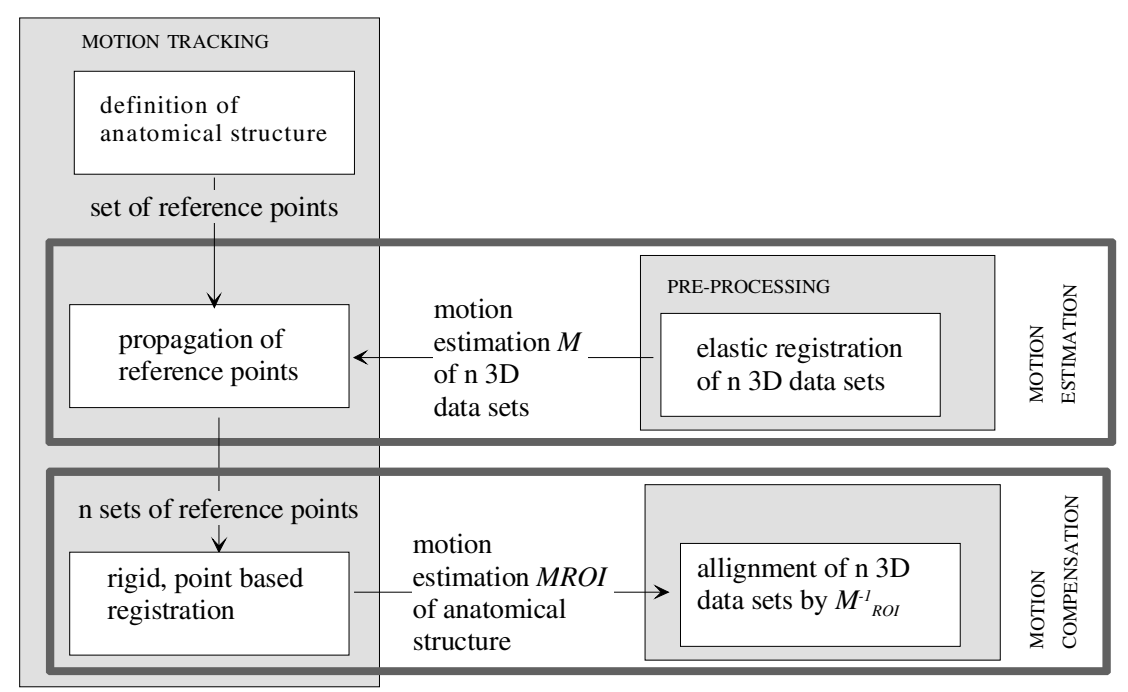

Fig. 2. Methods of image post-processing for fixation of an anatomical structure 


\subsection{Image Post-processing}

The essential part of the proposed kinematic workstation is motion tracking and motion compensation, which is realized by two different registration approaches (see Fig. 2).

\subsubsection{Motion Estimation}

Motion estimation of the entire kinematic 3D images is used to propagate the user defined reference points (see Section 2.1.2). The motion estimation is calculated by an elastic volumetric grey-value registration method based on a B-spline representation $[5,6]$. A mesh of control points with uniform spacing in each direction defines the transformation of the image. As cost function the sum of squared differences (SSD) over the total number of voxels is selected because the image contrast does not significantly change in the series. The optimization routine then determines from a set of admissible displacements a transformation, which minimizes this cost function. The optimization needs special attention, since the large number of parameters involved may result in high computational costs. We therefore choose an iterative LevenbergMarquardt method. Furthermore the B-spline registration is embedded into a multiscale approach employing both an image pyramid and a parameter pyramid of three to four levels. The multi-scale approach provides additional regularization and an increased capture range, and speeds up the overall algorithm.

\subsubsection{Motion Compensation}

Motion compensation is used to keep the user-defined anatomical region fixed in the viewing plane. Therefore the rotational and translational component of the region's motion has to be estimated, based on the selected and propagated set of reference points. Due to the propagation, a correspondence between all sets of reference points has been installed. Thus a rigid, point-based registration approach can be used, with the aim to estimate the motion of consecutive sets of reference points. Following Arun et.al. [4] the motion can be estimated by minimizing the least square distance of corresponding points of two 3D point sets. Using a singular value decomposition (SVD), the rotational matrix and based on it the translational vector can be calculated.

Since the user is not restricted in the selection of reference points these do not necessarily follow the same motion trajectories through the kinematic data sets. This might be desired in order to stabilize soft tissue structures like ligaments, but for reference point sets completely placed on solid structures like bones the point set should move in parallel. Therefore the SVD error provides an easy control value to verify quantitatively the reliability of the registration results as well as to check for misplaced reference points.

\section{Result}

\subsection{Viewing Component}

Our approach has been tested for different 4D kinematic MR data sets of the knee, the shoulder and the foot. The MR datasets were obtained using T1-weighted 3D gradient echo sequences for 7 different positions of the studied joint. Various anatomical objects within these data sets have been selected by (clinical) users, like the femur, the tibia, and the patella of the knee images, the scapula, and the tendon of the M. su- 
praspinalis of the shoulder images, and the tibia, fibula and talus in foot images. In all cases, the defined objects are fixed sufficiently, preventing in-plane and through-plane motion. The fixation of the femur is depicted in Fig. 3. The position of the distal part of the femur and the orientation of the femur's shaft remain the same during the movement from the fully stretched (Fig. 3 A) to the maximally inflected knee (Fig. 3 C).
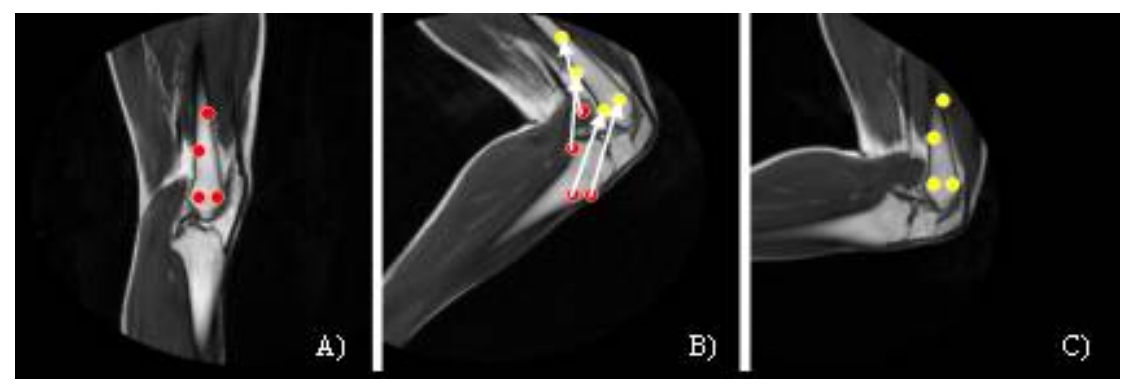

Fig. 3. A) Reference points are placed in the first frame. B) The motion of reference points is estimated from pre-computed elastic registration. C) The viewing plane is adapted to remove motion of reference points.

Following any user interaction, e.g. changing the viewing perspective or redefining the anatomy of interest, a tracked kinematic sequence is calculated instantaneously from the 4D MR data set, the user-defined reference points, and the tracking of these points via the stored elastic registration. This enables the clinical user to explore the kinematic data in real time, and allows the inspection of complex anatomical structures that have never been seen in (stabilized) motion before.

\subsection{Image Post-processing}

The quality of the automatic propagation of reference points by the B-spline registration algorithm is evaluated. Ideally, a reference point that has been propagated by motion estimation $\mathrm{M}_{\mathrm{i}, \mathrm{i}+1}$ from image $\mathrm{i}$ to image $\mathrm{i}+1$ and subsequently has been propagated back from image $i+1$ to $i$ by motion estimation $M_{i+1, i}$ will be located at its initial starting position. Thus the Euclidian distance of the original position of each reference point and its position can determine the consistency of the propagation after its propagation.
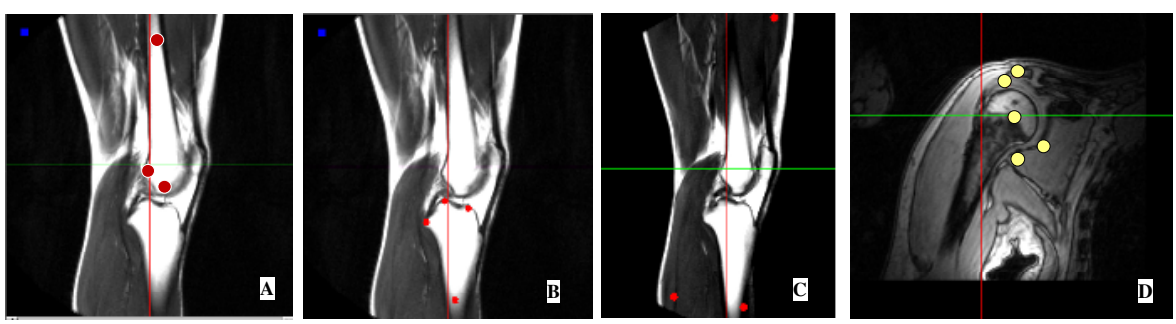

Fig. 4. Reference points of A) the femur, B) tibia, C) a non-rigid structure and D) the shoulder 
Table 1 shows the mean distances of different sets of reference points propagated between consecutive 3D images. The sets of reference points used in the knee and shoulder images are shown in Figure 4.

Table 1. Mean distance of sets of reference points in consecutive 3D images defining the femur, the tibia and the shoulder

\begin{tabular}{lllllll}
\hline Mean Distance & $1-2$ & $2-3$ & $3-4$ & $4-5$ & $5-6$ & $6-7$ \\
\hline Femur & $1.9 \mathrm{~mm}$ & $0.7 \mathrm{~mm}$ & $0.4 \mathrm{~mm}$ & $0.8 \mathrm{~mm}$ & $0.9 \mathrm{~mm}$ & $2.1 \mathrm{~mm}$ \\
Tibia & $1.2 \mathrm{~mm}$ & $0.3 \mathrm{~mm}$ & $0.4 \mathrm{~mm}$ & $0.5 \mathrm{~mm}$ & $1.2 \mathrm{~mm}$ & $0.8 \mathrm{~mm}$ \\
Shoulder & $0.8 \mathrm{~mm}$ & $0.3 \mathrm{~mm}$ & $0.2 \mathrm{~mm}$ & $1.9 \mathrm{~mm}$ & $1.0 \mathrm{~mm}$ & $0.5 \mathrm{~mm}$ \\
\hline
\end{tabular}

Another crucial step is the definition of the anatomical object or region of interest by the user. The residual error of the motion compensation is minimal at the centre and linearly grows with the distance from the centre of the mass of all reference points. At least 3 non-collinear points are required to define an object, where the points should be distributed evenly at its borders. However, we have experienced that (re-) defining an object by clicking is very intuitive for clinical experts, especially if feedback on the result is provided immediately.

In addition to the immediate visual feedback provided to the user table 3 shows the mean distance of reference points propagated using the SVD result versus using the registration parameter at their individual location. This value gives a quantitative measurement of the rigidness of the selected structure given a robust image registration. As expected from visual inspection table 3 shows small mean distances for rigid structures, whereas fixation of non-rigid regions leads to significant distances.

Table 2. Singular value decomposition error

\begin{tabular}{lllllll}
\hline Mean Distance & $1-2$ & $2-3$ & $3-4$ & $4-5$ & $5-6$ & $6-7$ \\
\hline Knee & & & & & & \\
\hline femur & $0.8 \mathrm{~mm}$ & $0.4 \mathrm{~mm}$ & $0.3 \mathrm{~mm}$ & $0.3 \mathrm{~mm}$ & $0.3 \mathrm{~mm}$ & $0.7 \mathrm{~mm}$ \\
tibia & $0.6 \mathrm{~mm}$ & $0.4 \mathrm{~mm}$ & $0.3 \mathrm{~mm}$ & $0.5 \mathrm{~mm}$ & $0.5 \mathrm{~mm}$ & $0.4 \mathrm{~mm}$ \\
patella & $0.4 \mathrm{~mm}$ & $0.4 \mathrm{~mm}$ & $0.3 \mathrm{~mm}$ & $0.2 \mathrm{~mm}$ & $0.4 \mathrm{~mm}$ & $0.1 \mathrm{~mm}$ \\
$\quad$ non-rigid region & $4.4 \mathrm{~mm}$ & $2.6 \mathrm{~mm}$ & $2.9 \mathrm{~mm}$ & $5.5 \mathrm{~mm}$ & $14.2 \mathrm{~mm}$ & $12.1 \mathrm{~mm}$ \\
$\begin{array}{l}\text { Shoulder } \\
\text { ligament }\end{array}$ & $1.2 \mathrm{~mm}$ & $0.5 \mathrm{~mm}$ & $0.8 \mathrm{~mm}$ & $3.7 \mathrm{~mm}$ & $2.2 \mathrm{~mm}$ & $1.8 \mathrm{~mm}$ \\
\hline
\end{tabular}

The B-spline registration has been embedded into a multi-scale approach. The image and mesh resolution is given by Table 2 . The original image size of the femur and tibia is $400 \times 400 \times 180 \mathrm{~mm}^{3}$ and the image size of the shoulder is $256 \mathrm{x} 256 \mathrm{x}$ $150 \mathrm{~mm}^{3}$. 
Table 3. Multiscale resolution of image and parameter space

\begin{tabular}{lll}
\hline Resolution & Image $\left[\right.$ voxel $\left.^{3}\right]$ & Mesh [control point \\
\hline Femur and Tibia & $36 \times 36 \times 16$ & $2 \times 2 \times 2$ \\
& $73 \times 73 \times 32$ & $4 \times 4 \times 4$ \\
\multirow{3}{*}{ Shoulder } & $256 \times 256 \times 36$ & $8 \times 8 \times 8$ \\
& $62 \times 62 \times 36$ & $2 \times 2 \times 2$ \\
& $124 \times 124 \times 72$ & $4 \times 4 \times 4$ \\
& $256 \times 256 \times 100$ & $8 \times 8 \times 8$ \\
& & $16 \times 16 \times 16$ \\
\hline
\end{tabular}

\section{Conclusion}

In conclusion, we have developed a workstation that facilitates viewing of 4D kinematic data sets. It allows to view in real-time any user defined anatomical structure from any viewing perspective. Smoothly displaying the movement in a cine-loop is realized by image post processing, fixing any user defined anatomical structures after image acquisition. Unrestricted from any pre-defined viewing perspective the clinical expert is enabled to examine any (fixed) anatomical structure during the movement of the entire joint in a 4D kinematic study.

\section{References}

1. F.G. Shellock, J.H. Mink, A.L. Deutsch, T.K.F. Foo. Kinematic MR imaging of patellafemoral joint: comparison of passive positioning and active movement techniques. Radiol. vol.184, pp.574-577, 1992.

2. U.H. Melchert, C. Schröder, J. BrossMann, C. Muhle. Motion-Triggered Cine MR Imaging of Active Joint Movement. MRI vol.10, pp.457-460, 1992.

3. D.K. Hodge, et.al. Dynamic MR imaging and testing in glenohumeral instability. JMRI vol.13, pp.748-756, 2001.

4. 4.K.S. Arun, T.S. Huang, S.D. Blostein. Last squares fitting of two 3D point sets. IEEE Trans. Pat. Anal. Mach. Intell., vol 9, pp. 698-700, 1987.

5. S. Kabus, et.al.., B-spline registration of 3D images with Levenberg-Marquardt optimization. SPIE Medical Imaging, 2004.

6. D. Rueckert, L.I .Sonoda. D.L. Hill, M.O. Leach, and D.J. Hawkes. Nonrigid registration using free-form deformations: Application to breast MR. IEEE Transactions on Medical Image Processing, 18(8): 712-721, 1999. 